\title{
The Pennington Lecture
}

\section{Chronic idiopathic intestinal pseudo-obstruction: the need for a multidisciplinary approach to management}

\author{
D. B. A. Silk \\ Department of Gastroenterology and Nutrition, Central Middlesex Hospital and Division of Surgery, \\ Anaesthetics and Intensive Care, Imperial College, London, UK
}

\begin{abstract}
At the outset of the research programme into irritable bowel syndrome (IBS) it was perceived that there was a need to develop a symptom-based classification for the patients. Four groups of patients were identified, those with spastic colon syndrome, diarrhoea-predominant spastic colon syndrome, functional diarrhoea and midgut dysmotility. While working with outpatients with IBS it was noted how some of them had suffered symptoms for many years; specifically, a group of patients satisfying the criteria for midgut dysmotility had also suffered from particularly severe and intractable intestinal symptoms. These patients underwent $24 \mathrm{~h}$ ambulatory studies of small intestinal motility and the majority were found to have manometric features of chronic idiopathic intestinal pseudo-obstruction (CIIP). To characterise the cause, laparoscopic full-thickness small intestine and colonic biopsies have been obtained in forty-five of the latter group of patients. Of these patients $58 \%$ have been found to have complete or partial deficiency of $\alpha$-actin epitope staining in the inner circular layer of small intestinal smooth muscle. This deficiency is believed to represent an important biomarker rather than the cause of CIIP, since $\alpha$-actin epitope deficiency has been observed in association with enteric neuropathy and myopathies. In relation to the management of CIIP patients, a multidisciplinary model is proposed incorporating management of co-morbid psychological and psychiatric pathology, abdominal and musculoskeletal pain, fatigue, urological symptoms and nutrition. A six-stage nutritional management plan for these patients is presented.
\end{abstract}

Chronic idiopathic intestinal pseudo-obstruction: Irritable bowel syndrome: Symptom-based classification: Multidisciplinary management model: Nutritional management plan

It was a privilege to deliver the second Pennington Lecture at the 12th Annual Meeting of the British Association for Parenteral and Enteral Nutrition (BAPEN). The author's tenure as BAPEN Chairman ended in 1999, and it was clear, after 5 years in the Chair, that what was needed to carry BAPEN forward was someone who had a broad experience of both academic and clinical aspects of Clinical Nutrition and a person who at the same time was used to a multidisciplinary approach to the subject and would command the respect of all the parties involved. Chris Pennington, of course, was the overwhelmingly obvious choice and everyone would agree that Chris' greatest contribution to
BAPEN was as its Chairman. Following his untimely death in May 2002, the officers and Council of BAPEN wrote 'BAPEN as well as the field of clinical nutrition has lost a leader with vision, a colleague with compassion, a mentor with time and a friend who cared'. The ensuing article is dedicated to him, and he would have wished his lecturers to look into the future, not dwell in the past, and present new rather than old research data. It is hoped that the subject matter presented satisfies this brief.

The 'system' in many academic medical units is that clinical and academic research is blended with clinical responsibilities and this situation has certainly, over the

\footnotetext{
Abbreviations: BAPEN, British Association for Parenteral and Enteral Nutrition; CIIP, chronic idiopathic intestinal pseudo-obstruction; IBS, irritable bowel syndrome; MMC, migrating motor complex. 
years, existed in the Unit at Central Middlesex Hospital. Indeed, at a time when complex intubation techniques were being developed to study small intestinal transit and motility during ileal infusion (Spiller et al. 1984, 1988), and to simultaneously monitor small intestinal motility and quantify colonic inflow volumes (Raimundo et al. 1988, 1990), as well as undertaking regional colonic perfusion studies (Bowling et al. 1993), all the clinicians involved in the research were also working in busy gastroenterology outpatient clinics in which more than half the patients seen had a functional gastrointestinal disorder, the commonest of which was the irritable bowel syndrome (IBS; Harvey et al. 1983; Weber \& McCullum, 1992).

It seemed that gastrointestinal physiologists were being asked to see a largely heterogeneous group of patients, different groups of patients having different symptoms referable to different sites of the mid- and hindgut, and that as a generalisation a single mode of therapy (e.g. colofac (Solvay Healthcare Ltd, Southampton, Hants., UK) plus fibre supplements) was highly unlikely to represent an across-the-board treatment approach.

More than 10 years ago, therefore, categorisation of patients (most of whom were assumed to have IBS) into different groups or variants depending on common symptom complexes was begun (Silk, 1995a,b). Although the symptom-based classification that is being used is based on similar set of premises to those that subsequently formed the rationale for the Rome II classification system (Drossman, 1999), it is not in complete agreement with that of Rome II (Cole et al. 2002; Silk, 2003).

Specifically, the author's group and others (Camilleri $\&$ Choi, 1997) have had concerns about the particularly restrictive nature of the Manning Criteria (Manning et al. 1978) to define IBS, as neither the functional diarrhoea defined by Heaton (1984) nor the pain-gas-bloat symptomatic subgroup described by Camilleri and colleagues (Camilleri \& Prather, 1992; Camilleri \& Choi, 1997) would be identified using the Rome II criteria (Thompson et al. 1999). Accordingly, a system has been developed of categorising patients with IBS into four groups according to clinical features. These groups are: spastic colon syndrome; functional diarrhoea; midgut dysmotility; diarrhoeapredominant spastic colon syndrome (Cole et al. 2002).

\section{Spastic colon syndrome}

The chosen criteria are based on those of Manning et al. (1978) and the clinical diagnosis depends on four or more of the following symptoms coexisting: (a) visible abdominal distension; (b) pain relieved by bowel action; (c) more frequent stools with the onset of pain; (d) looser stools with the onset of pain; (e) rectal passage of mucus; (f) sensation of incomplete evacuation.

\section{Functional diarrhoea}

The chosen criteria are passage based on symptoms described by Heaton (1984), i.e. stools in rapid succession in the morning, then either normal or no further defaecation, plus two or more of: (a) first stool formed, the later ones loose; (b) urgency of defaecation; (c) anxiety about bowel function (e.g. travelling); (d) exhaustion after the 'morning rush'.

\section{Midgut dysmotility}

Recent experimental evidence, as well as clinical experience, has suggested that there is a group of patients with functional gastrointestinal disorders in whom symptoms arise as a consequence of abnormal small intestinal function. From the clinical standpoint these patients may present with diffuse abdominal pain, often postprandially, bloating not restricted to the upper abdomen, postprandial fullness, 'trapped wind', nausea and anorexia. These symptoms occur in the absence of any alteration of bowel habit and are not relieved by bowel evacuation. Experimental evidence favouring the inclusion of patients with these symptoms is that patients with IBS have been shown to have abnormal small intestinal motility (Schmidt et al. 1996; Small et al. 1997) that may be associated with the symptoms (Kumar \& Wingate, 1985; Kellow \& Phillips, 1987). Pain in IBS can be reproduced by inflating balloons in the small intestine (Moriarty \& Dawson, 1982), and patients with IBS have been shown to have selective dysfunction of mechano-sensitive small intestinal afferents (Accarino et al. 1995). Impaired ileo-caecal valve function in patients with IBS presenting with right-sided abdominal pain and bloating has also been demonstrated (Trotman \& Price, 1986).

The chosen criteria for inclusion into this midgut dysmotility group are: diffuse abdominal discomfort or pain not relieved by bowel action, not associated with the passage of more frequent or looser stools and not accompanied by the passage of mucus per rectum or by a sense of incomplete evacuation, and two of the following: (a) diffuse abdominal bloating; (b) postprandial fullness or early satiety; (c) nausea; (d) anorexia.

Patients categorised to the midgut dysmotility group according to these criteria are likely to present a similar clinical picture to the 'bloated IBS' described by Trotman \& Price (1986) and those with pain-gas-bloat described by Camilleri and colleagues (Camilleri \& Prather, 1992; Camilleri \& Choi, 1997).

\section{Diarrhoea-predominant spastic colon syndrome}

During the course of early phases of the work, it became apparent that there existed a further group of patients who had some of the clinical features of both spastic colon syndrome and functional diarrhoea but did not satisfy the inclusion criteria for either group. These patients have been grouped together to form a diarrhoea-predominant spastic colon group (Cole et al. 2002), the predominant symptoms being abdominal pain relieved by defaecation 'rushes' of diarrhoea and incomplete evacuation. This group of patients is similar to the diarrhoea-predominant IBS group described in the Rome II criteria (Thompson et al. 1999).

Studies have been undertaken to characterise fasting and postprandial distal colonic motility in those patients 
(Cole et al. 2002). Marked reductions in distal colonic motility compared with controls have been noted in the two groups of patients with diarrhoea (functional diarrhoea and diarrhoea-predominant spastic colon syndrome). In contrast, patients with spastic colon syndrome have more vigorous motility than controls. Importantly, the patients with midgut dysmotility have normal distal colonic motility, emphasising how the dysmotility in this group of patients with IBS is likely to reside in the midgut (Cole et al. 2002).

\section{Severe midgut dysmotility}

While working with patients with IBS there was recognition that some patients have suffered symptoms for many years. Two large surveys to look into this situation further have been conducted via the IBS Bulletin (Silk, 1998, 2001), which is a quarterly publication provided as a service to patients. At the time of the surveys there were approximately 4000 subscribers. The mean duration of symptoms in the surveyed patients with IBS was found to be $\leq 16$ years (Silk, 2001).

Focusing further on the chronicity problem, there seemed to be a subgroup of patients satisfying the criteria for inclusion into the midgut dysmotility variant of IBS who not only had very-long-standing symptoms, but whose abdominal pain was very severe, intractable, postprandial and, importantly, nocturnal. Some patients seemed to have normal bowel function, whereas others seemed to suffer from particularly severe slow-transit constipation (see Table 1). In order to characterise the small-bowel motility patterns that might be giving rise to symptoms in these patients, $24 \mathrm{~h}$ ambulatory studies of small intestinal motility have been undertaken. Standard manometric techniques (Stanghellini et al. 1987; Raimundo et al. 1996; Camilleri et al. 1998) were employed. A pattern of abnormal small intestinal motility characterised by aberrant configuration of the phase III of the migrating motor complex (MMC), abnormal propagation of the MMC (Fig. 1) or absence of the phase III of the MMC emerged. In addition, abnormal bursts of non-propagated activity (Fig. 2), discreet cluster contractions and sustained uncoordinated phasic activity were also identified. Small intestinal manometry has now been undertaken in over fifty patients with severe midgut dysmotility, and the abnormalities seen in the patients all conform to this description. What is striking about the findings is that they are the characteristic manometric abnormalities described in patients with chronic idiopathic intestinal pseudo-obstruction (CIIP; Stanghellini et al. 1987); findings, in turn, that are generally considered predictive of enteric neuropathy as the dominant

Table 1. Patients with severe midgut dysmotility variant of irritable bowel syndrome

Long-standing symptoms

Severe pain

Severe to intractable pain

Nocturnal pain

With or without severe constipation

All standard investigations, including $\mathrm{Ba}$ follow-through, normal

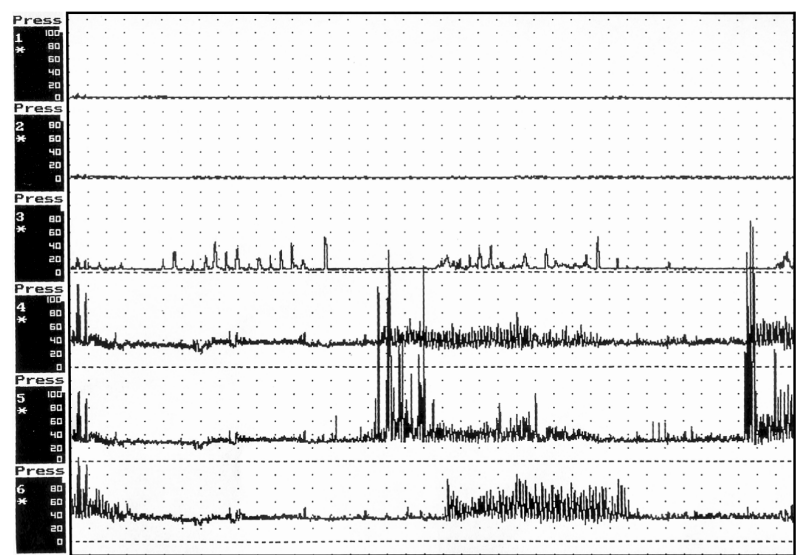

Fig. 1. Motility recording from the small intestine of a patient with severe midgut dysmotility variant of irritable bowel syndrome. There were four sensors (3-6) sited down the small intestine. The top (sensor 3 ) was positioned in the proximal small intestine. The phase III activity front of the migrating motor complex (MMC) should propagate distally through the small intestine, i.e. more to the right along the horizontal axis from sites 4 to 6 . This pattern represents an abnormally-propagated phase II MMC.

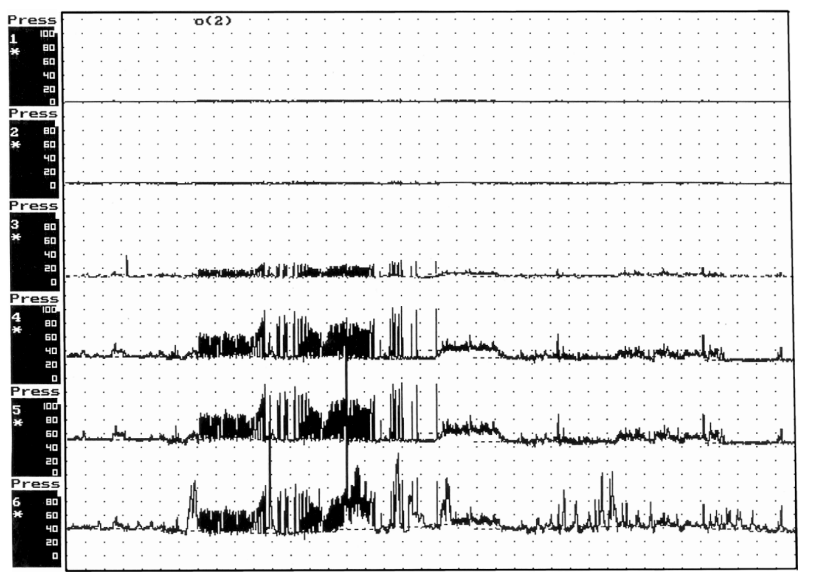

Fig. 2. Motility recording from the small intestine of a patient with severe midgut dysmotility variant of irritable bowel syndrome. There were four sensors (3-6) sited down the small intestine. The top (sensor 3 ) was positioned in the proximal small intestine. There is an abnormal burst of high-pressure activity recorded by sensors 4,5 and 6 that does not propagate distally through the small intestine.

lesion (Stanghellini et al. 1987; Quigley et al. 1997; Camilleri et al. 1998; Wingate et al. 2002; Scott et al. 2003).

With the aim of determining the cause of the apparent CIIP, laparoscopic full-thickness biopsies of the jejunum and colon have been obtained in a group of these patients using an end gastrointestinal anastamotic stapling device (mean operating time $13 \mathrm{~min}$, Knowles et al. 2004). To date, biopsies have been obtained in forty-five of these patients (Table 2). The details of the histopathological techniques used to stain biopsy sections have been described (Knowles et al. 2004). The most striking finding is the absence in fourteen $(31 \cdot 1 \%)$ of the patients of jejunal smooth-muscle $\alpha$-actin immunostaining in the circular 
Table 2. Histological findings in chronic idiopathic intestinal pseudo-obstruction ( $n$ 53)

\begin{tabular}{|c|c|}
\hline Not biopsied & 8 \\
\hline Normal biopsies & 9 \\
\hline$\alpha$-Actin epitope deficiency: Alone & 22 \\
\hline +Enteric neuropathy & 4 \\
\hline Normal $\alpha$-actin staining: Enteric neuropathy & 2 \\
\hline Enteric vasculitis & 1 \\
\hline Enteric myositis & 1 \\
\hline Colonic myopathy & 1 \\
\hline Misplaced myenteric plexus & 1 \\
\hline
\end{tabular}

muscle layer, with the exception of its innermost layer adjacent to the submucosa, where the smooth muscle cells stain strongly positive. In twelve $(27 \cdot 7 \%)$ further cases there is incomplete (partial) loss of staining in the circular muscle layer. The interest in the deficiency of the jejunal circular muscle $\alpha$-actin as a possible biochemical cause of CIIP stems originally from a single cause report of its deficiency in a 48-year-old female with clinical radiological and physiological features of CIIP (Smith et al. 1992).

Initially, it seemed reasonable to believe that the biochemical abnormalities in smooth-muscle $\alpha$-actin might be responsible for causing the abnormalities of motility and symptoms in those patients with CIIP who had the disorder. However, disturbances of manometry observed in the patients with CIIP (predominantly of the phase III MMC with or without abnormal additional non-propulsive activities) are thought to be indicative of a motor programming abnormality, i.e. caused by neural dysfunction rather than an intrinsic myopathic disturbance in which a pattern of generalised hypomotility is often observed (Camilleri et al. 1998). The subsequent discovery of coexisting degenerative or inflammatory neuropathic abnormalities in the series in four patients with $\alpha$-actin deficiency has prompted a reconsideration of the functional importance of the finding of special alterations in smooth-muscle $\alpha$-actin staining in the context of its pathophysiological role. The subsequent description in the literature of two further patients with a deficiency of staining of smooth-muscle $\alpha$-actin who had coexisting enteric myopathy (Moore et al. 2002) and an inflammatory myositis (Ruuska et al. 2002) provides added support that the alteration in staining in jejunal circular muscle in CIIP patients may not in itself be causative or specific to the disorder. Conversely, it is equally clear that the abnormality represents a useful biomarker of CIIP, especially in the absence of any other structural abnormality (Knowles et al. 2004). Whether or not these patients with CIIP who have $\alpha$-actin epitope deficiency have a common underlying disorder such as a channellopathy remains to be seen.

\section{Management}

Management of the patient with CIIP necessarily has to be considered on an individual basis and there are often multi-system components to the diagnosis (pain, psychiatric, fatigue, musculoskeletal, urological and nutritional) and a multidisciplinary approach to management may therefore be required.

\section{Psychiatric disorders}

Almost all the patients with CIIP discussed here were referred for assessment and management of their functional gastrointestinal disorders (most commonly IBS). Many patients had sought advice from or been referred to psychiatrists before diagnosis. In view of the chronicity and severity of their symptoms it is not surprising that a variety of diagnoses had been offered before diagnosis, including anxiety, depression, somatisation, abnormal illness attitude, poor social attitude and ineffective coping attitudes. Based on current knowledge of how biopsychosocial factors interact and modulate experiences of pain in general (Holdcroft \& Power, 2003) and other symptoms of functional gastrointestinal disorders (Drossman, 1999), the co-morbid psychopathological components are very likely to affect the clinical picture of CIIP. The skills of liaison psychiatrists and clinical psychologists with an interest in functional gastrointestinal disorders have been invaluable in the management of these patients. Some of the patients with severe co-morbid psychopathology have required and done well with electro-convulsive therapy. Tricyclic antidepressants should be used with caution in these patients, as they have been shown to prolong both oro-caecal and whole-gut transit times (Gorard et al. 1994), at least in part by reducing the velocity of the phase III of the MMC (Gorard et al. 1995). The resultant constipation can be troublesome, particularly if pre-existing. Clinical experience has indicated that selective serotonin noradrenaline re-uptake inhibitors may also cause constipation, and the use of selective serotonin re-uptake inhibitors (particularly paroxetine) seems to be more beneficial to these patients. It is interesting that paroxetine has been shown to have a prokinetic effect on small bowel motility, shortening oro-caecal transit time and increasing the velocity of the phase III of the MMC (Gorard et al. 1994, 1995).

\section{Pain}

Abdominal pain has been one of the major presenting symptoms in the majority of the CIIP patients. As the manometric features of the patients are more in keeping with an enteric neuropathy than myopathy the experiences are similar to those of Mann et al. (1997), all of whose adult patients with enteric neuropathy had pain as a 'main' symptom.

The pain in the patients with CIIP is likely to arise either on account of the abnormal and exaggerated motility or as a consequence of visceral hyperalgesia, or a combination of the two mechanisms, the magnitude being influenced by coexisting brain-gut axis dysrhythmia (Drossman, 1999; Castle et al. 2004). Attempts to normalise the small intestinal dysmotility, thereby improving the pain in patients with CIIP, have met with little success, and in agreement with other findings the use of erythromycin, maxolon, domperidone, cisapride and octreotide have been largely ineffective (Dyer et al. 1969; Lipton \& Knauer, 1977; Camilleri et al. 1986, 1989; Verne et al. 1995; Mann et al. 1997). The exception relates to patients with CIPP with associated mixed connective tissue disorders and thyroid 
disease. In these patients the treatment of the underlying disease has resulted in a marked improvement of the pharmacologically-responsive intestinal pain resulting from CIIP, which has previously been described in patients with systemic lupus erythematosis (Perlemuter et al. 1998). One of the patients, who is experiencing severe intestinal pain as a result of CIIP, has had a temporary remission when receiving chemotherapy for acute myeloid leukaemia. Whether the intestinal dysmotility in the patients can be reversed by the use of either a serotonin type 4 receptor agonist or intestinal pacing remains to be determined.

Experience indicates that the management of intestinal or visceral pain in patients with CIIP is very difficult. It is preferred, of course, that patients should not be on opiates at the time of referral, because the majority of those on opiates are addicted. The key to success in these patients is the use of non-opioid analgesia with full psychological and psychiatric support whenever needed. Although nonopioid analgesia is not renowned as a means of controlling visceral pain, it can be successfully used in patients with CIIP. In those patients treated this way it has certainly been the case, with only twelve patients having to resort to the use of opioid analgesia, of whom six patients have been addicted at one stage at least. Apart from the usual sequalae of opioid addiction, the onset of severe extraintestinal infections has been noted in three of the patients with CIIP addicted to opioids (infective arthritis of the knee, infective sacroileitis bacteraemia and multiorgan failure). As pointed out (Richards et al. 1997) opiate dependence predicts a poor outcome for patients receiving home parenteral nutrition, so this form of nutritional support has not been encouraged in the patients with CIIP who are opioid dependent.

\section{Fatigue}

Of a series of 100 consecutive patients with IBS $60 \%$ have reported 'constant tiredness' (Whorwell et al. 1986b). A substantial number of the current series of patients with CIIP have also complained of fatigue and tiredness. At present, this aspect of the symptomatology has not been properly characterised and it is planned to determine whether the tiredness and fatigue suffered by the patients satisfies the 1994 Centers for Disease Control definition of idiopathic chronic fatigue, as originally proposed by the International Chronic Fatigue Syndrome Study Groups (Fukuda et al. 1994).

\section{Musculoskeletal pain}

Medically-unexplained chronic widespread musculoskeletal pain has a community prevalence of $11-13 \%$ in the UK (Croft et al. 1993; Macfarlane et al. 1999). Fibromyalgia, the severe end of the spectrum, comprises musculoskeletal pain in association with multiple tender points (Wolfe et al. 1990). A number of the patients with CIIP have been formally diagnosed as having fibromyalgia, and indeed in two patients the symptoms arising are their most clinically important. It is planned to formally assess the whole series of patients in the near future.

\section{Urological symptoms}

Previous studies have shown that patients with IBS normally have a higher incidence of urinary symptoms than matched controls (Whorwell et al. 1986b), but on formal urodynamic studies the incidence of bladder dysfunction is $50 \%$ (Whorwell et al. 1986a), with detrusor muscle instability being the commonest cause (Whorwell et al. 1986a). The Unit patients with CIIP also commonly have urinary symptoms, and indeed a number of them have 'neurogenic' bladders and have to self catheterise. A number of the patients on formal testing have a complete failure of detrusor muscle function, suggesting the possibility that in these patients there is a common pathology affecting the smooth muscle of the small intestine and bladder. Further work is needed, and indeed is in hand, to fully characterise the urological pathophysiology in these patients.

\section{Nutritional management}

The nutritional status of the patients with CIIP is variable, ranging from overnutrition in two patients to undernutrition. Although the nutritional status of the patients has not yet been formally assessed, the majority of them seem to be able to maintain normal or near-normal nutritional status; it is those who became malnourished that present the greatest challenge. Malnutrition arises in this group of patients because their abdominal symptoms, (pain, fullness, distension, bloating, perception of build up of wind) are exacerbated or triggered by eating and, not unnaturally, this situation leads in some patients to a chronic reduction in nutritional intake. Moreover, many of the patients have perceived food intolerances with what they consider to be food-provoking symptoms. It is suggested that there may be an important link between psychological factors and the development of these foodrelated symptoms, and that in some patients the foodrelated symptoms develop as a consequence of disturbed intestinal motility that is mediated by a psychologicallyinduced corticotrophin-releasing hormone stress response (Fukudo et al. 1998). However, the majority of the patients with CIIP have abnormal postprandial motility that may be triggered not by specific food items but by eating. A consistent observation made by the patients themselves is that they are able to better tolerate food by eating small frequent meals. This situation suggests that if lumen nutrient concentrations or loads (concentration $\times$ rate) are kept below a defined threshold the abnormal postprandial motility may not be stimulated or triggered. Further studies are required to confirm this relationship. However, it has been observed that if the first stage of nutritional management (normal eating pattern; Table 3 ) cannot be managed then patients will progress to stages 2 and 3 , consuming frequent small meals with or without oral nutritional supplements under dietetic supervision. If nutritional status cannot be maintained in this way some form of artificial nutrition support then becomes a necessity.

Clinical experience has indicated that enteral feeding by percutaneous endoscopic gastrostomy tube is rarely tolerated by the patients with CIIP who reach this stage 
Table 3. Stages of nutritional management of patients with chronic idiopathic intestinal pseudo-obstruction

\begin{tabular}{ll}
\hline Stage & \\
\hline 1 & Normal eating pattern \\
2 & Frequent small meals \\
3 & Frequent small meals +oral nutritional supplements \\
4 & Naso-gastric enteral tube feeding \\
5 & PEG tube enteral feeding \\
6 & Naso-jejunal enteral tube feeding \\
6 & Needle catheter jejunostomy feeding \\
& Total parenteral nutrition \\
\hline
\end{tabular}

PEG, percutaneous endoscopic gastrostomy.

of nutritional management. In the Unit the potential of enteral feeding by percutaneous endoscopic gastrostomy tube as a means of artificial nutritional support is tested by instituting naso-gastric tube feeding (stage 4 ; Table 3 ). If this method of feeding is poorly tolerated, enteral feeding by percutaneous endoscopic gastrostomy tube is not considered as an option. As the small intestinal motility responses to naso-gastric and naso-duodenal tube feeding differ, with abnormal motility patterns being noted during naso-gastric tube feeding (Raimundo et al. 1988, 1990), the efficacy of post-pyloric duodenal or jejunal (post ligament of trietz) feeding administered via nasoenteral feeding tubes has been investigated in some of the patients (stage 5; Table 3). Some patients have been able to tolerate this route of feeding, and in these patients needle-catheter jejunostomy feeding tubes have then been sited. A few of the patients have been successfully fed for reasonably long periods of time ( $\geq 6$ years) via this route. To date, only one of the series of patients is failing to maintain nutritional status on needle-catheter jejunostomy feeding, and has been considered for home parenteral nutrition. Unfortunately, the patient has been opiate dependent, which means that the outlook for home parenteral nutrition is bad (Richards et al. 1997). The patient is, therefore, currently commencing a further trial of needle-catheter jejunostomy tube feeding. A number of the patients with CIIP have experienced a good deal of abdominal pain in association with their needle-catheter jejunostomy feeding tubes, and it is recognised, but not understood, that it may be possible to overcome unacceptable tube-related pain by re-siting it.

\section{Patient-focused multidisciplinary management}

It follows from the earlier discussion that the challenges to the management of the patients with CIIP can only be met by a multidisciplinary approach, and a model has been proposed (Fig. 3). All aspects of management are interlinked and no single area of management is exclusive of the others. Control of abdominal pain, for example, is linked to the management of nutrition status as well as management of musculoskeletal and fibromyalgic symptoms, urological symptoms, tiredness and fatigue, with a particularly important interconnecting area of management being that of the underlying psychological and psychiatric symptoms.

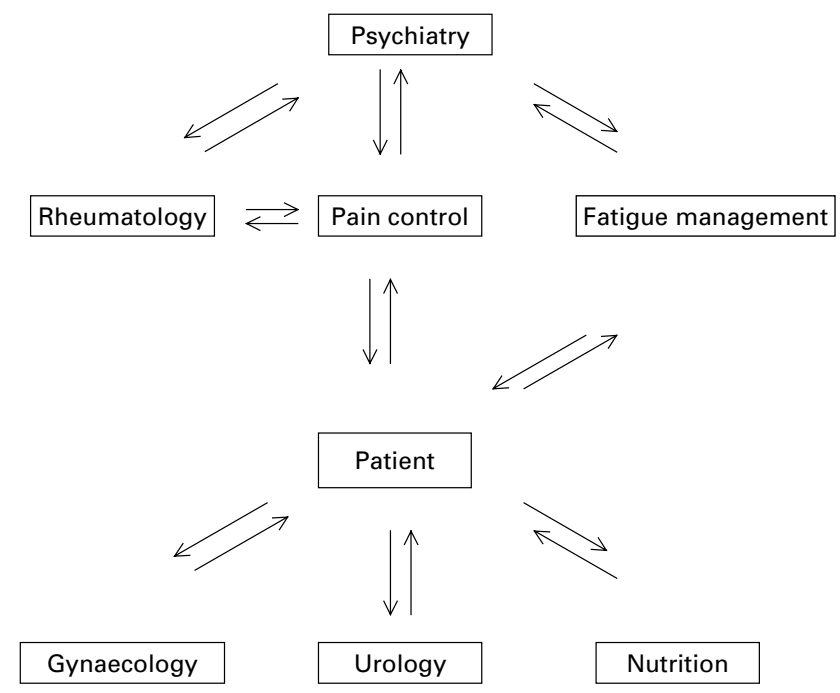

Fig. 3. The multidisciplinary approach to the management of chronic idiopathic intestinal pseudo-obstruction syndrome.

It is clear that the present and future management of the patients with CIIP will necessarily place an unreasonable financial burden on current resources. Unfortunately, CIIP is a poorly understood condition and a recent multidisciplinary application to the Department of Health for funding from the National Specialised Commissioning Advisory Group has been rejected and a re-submission discouraged. However, it is believed that when the enormity of the condition is fully realised by funding agencies, the patients will be able to receive the properly coordinated multidisciplinary care that they deserve.

\section{Acknowledgements}

I am greatly indebted Dr Gerard Libbey my psychiatric colleague and to all the research fellows, in particular Mr Simon Cole, Dr Adrian Claydon and Dr David Austin, who have supported my concepts of CIIP and who have helped with the diagnosis and management of the patients. Thanks also go to Dr Ana Raimundo who has undertaken almost all the small intestinal motility studies. I am particularly grateful to Mr Charles Knowles for his help, support and, most importantly, his intellectual input into the study. Professor Sir Ara Darzi's contribution in undertaking the laparoscopic biopsies has been pivotal and Professor Claire Fowler's contribution will lead to the full characterisation of the co-morbid urological pathology. None of my work would have come to fruition, however, had it not been for the involvement of Professor Jo Martin. Her gastrointestinal neuro-histopathological expertise has been second to none, and her enthusiasm for the study has kept us all at our work.

\section{References}

Accarino AM, Azpiroz F \& Malagelada J (1995) Selective dysfunction of mechanosensitive intestinal afferents in irritable bowel syndrome. Gastroenterology 108, 636-643. 
Bowling TE, Raimundo AH, Grimble GK \& Silk DB (1993) Reversal of short-chain fatty acids of colonic fluid secretion induced by enteral feeding. Lancet 342, 1266-1268.

Camilleri M, Brown ML \& Malagelada JR (1986) Impaired transit of chime in chronic intestinal pseudoobstruction: correction by cisapride. Gastroenterology 91, 619-626.

Camilleri M \& Choi M-G (1997) Review article: irritable bowel syndrome. Alimentary Pharmacology and Therapeutics 11, 3-15.

Camilleri M, Haslam WL, Parkman HP, Quigley EM \& Soffer E (1998) Measurement of gastrointestinal motility in the GI laboratory. Gastroenterology 115, 747-762.

Camilleri M, Malagelada JR, Abell TL, Brown ML, Hench V \& Zinsmeister AR (1989) Effect of six weeks of treatment with cisapride in gastroparesis and intestinal pseudo-obstruction. Gastroenterology 96, 704-712.

Camilleri M \& Prather CM (1992) The irritable bowel syndrome: mechanisms and a practical approach to management. Annals of Internal Medicine 116, 1001-1008.

Castle MZD, Silk DBA \& Libby GW (2004) The rationale for antidepressant therapy in functional gastrointestinal disorders. Alimentary Pharmacology and Therapeutics 19, 1-11.

Cole SJ, Duncan HD, Claydon AH, Austin D, Bowling TJ \& Silk DBA (2002) Distal colonic motor activity in four subgroups of patients with irritable bowel syndrome. Digestive Diseases and Sciences 47, 345-355.

Croft P, Rigby AS, Boswell R, Schollum J \& Silman A (1993) The prevalence of chronic widespread pain in the general population. Journal of Rheumatology 20, 710-713.

Drossman DA (1999) The functional gastrointestinal disorders and the Rome II process. Gut 45, Suppl. 11, 111-115.

Dyer NH, Dawson AM, Smith BF \& Todd IP (1969) Obstruction of bowel due to lesion in the myenteric plexus. British Medical Journal 1, 686-689.

Fukuda K, Strauss S, Hickie I, Sharpe M, Dobbins J \& Komaroff A (1994) The chronic fatigue syndrome; a comprehensive approach to its definition and study. International Chronic Fatigue Syndrome Study Group. Annals of Internal Medicine 121, 953-959.

Fukudo S, Nomura T \& Hongo M (1998) Impact of corticotrophin releasing hormone on gastrointestinal motility and adrenocorticotropic hormone in normal controls and patients with irritable bowel syndrome. Gut 42, 845-849.

Gorard DA, Libby GW \& Farthing MJ (1994) Influence of antidepressants on whole gut and orocaecal transit times in health and irritable bowel syndrome. Alimentary Pharmacology and Therapeutics $\mathbf{8}, 159-166$.

Gorard DA, Libby GW \& Farthing MJ (1995) Effect of a tricyclic antidepressant on small intestinal motility in health and diarrhoea-predominant irritable bowel syndrome. Digestive Diseases and Sciences 40, 86-95.

Harvey RF, Salih SY \& Read AE (1983) Organic and functional disorders in 2000 gastroenterology outpatients. Lancet $\mathbf{i}$, 632-634.

Heaton KW (1984) Irritable bowel syndrome. In Textbook of Gastroenterology, pp. 867-875 [IAD Bouchier, RN Allen, JHF Hodgson and MRB Keighley, editors]. London: Bailiere Tindall.

Holdcroft A \& Power I (2003) Management of pain. British Medical Journal 326, 635-639.

Kellow JE \& Phillips SF (1987) Altered small bowel motility in the irritable bowel syndrome is correlated with symptoms. Gastroenterology 92, 1885-1893.

Knowles CH, Silk DBA, Darzi A, Feakins R, Raimundo AH, Veress B, Crompton T, Lindberg G \& Martin JE (2004) Deranged smooth muscle alpha fetoprotein-actin: a biomarker of intestinal pseudo-obstruction: a controlled multinational case series. Gut (In the Press).
Kumar D \& Wingate DL (1985) The irritable bowel syndrome: a paroxysmal motor disorder. Lancet ii, 973-977.

Lipton AB \& Knauer CM (1977) Pseudo-obstruction of the bowel. Therapeutic trial of metoclopramide. American Journal of Digestive Diseases 22, 263-265.

Macfarlane GJ, Morris S, Hunt IM, Benjamin S, McBeth J, Papageorgiou AC \& Silman AJ (1999) Chronic widespread pain in the community: the influence of psychological symptoms and mental disorder on healthcare seeking behaviour. Journal of Rheumatology 26, 413-419.

Mann SD, Debinski HS \& Kamm MA (1997) Clinical characteristics of chronic idiopathic intestinal pseudo-obstruction in adults. Gut 41, 675-681.

Manning AP, Thompson WG, Heaton KW \& Morris AF (1978) Towards a positive diagnosis of the irritable bowel syndrome. British Medical Journal ii, 653-654.

Moore SW, Schneider JW \& Kaschula ROC (2002) Unusual variations of gastrointestinal smooth muscle abnormalities associated with chronic intestinal pseudo-obstruction. Pediatric Surgery International 18, 13-20.

Moriarty KJ \& Dawson AM (1982) Functional abdominal pain: further evidence that the whole gut is involved. British Medical Journal 284, 1670-1672.

Perlemuter G, Chaussade S, Wechsler B, Cacoub P, Dapiogny M, Kahan A, Godeau P \& Courturier D (1998) Chronic intestinal pseudo-obstruction in systemic lupus erythematosis. Gut $\mathbf{4 3}$ $117-122$.

Quigley EM, Deprez PH, Helstrom P, Husebye E, Soffer EE, Stangh V, Summers RW, Wilmer A \& Wingate DL (1997) Ambulatory intestinal manometry: a consensus report on its clinical role. Digestive Diseases and Sciences 42, 2395-2400.

Raimundo AH, Rogers J, Grimble G, Cahill E \& Silk DBA (1988) Colonic inflow and small bowel motility during intraduodenal enteral nutrition. Gut 29, A1469-A1470.

Raimundo AH, Rogers J \& Silk DB (1990) Is enteral feeding related diarrhoea initiated by an abnormal colonic response to intragastic diet infusion? Gut 31, A119.

Raimundo AH, Rogers J \& Silk DBA (1996) Prolonged ambulatory small intestinal and colonic motility monitoring: potential in irritable bowel syndrome - first report of prolonged ambulatory oesophageal, small intestinal and colonic motility in the same patient. European Journal of Gastroenterology and Hepatology 8, 81-84.

Richards DM, Scott NA, Shaffer JL \& Irving M (1997) Opiate and sedative dependence predicts a poor outcome for patients receiving home parenteral nutrition. Journal of Parenteral and Enteral Nutrition 21, 336-338.

Ruuska TH, Karikoski R, Smith VV \& Milla PJ (2002) Acquired myopathic intestinal pseudo-obstruction may be due to autoimmune enteric leiomyositis. Gastroenterology $\mathbf{1 2 2}$, 1133-1139.

Schmidt T, Hackelsberger N, Widmer R, Meisel C, Pfeiffer A \& Kaess H (1996) Ambulatory 24-hour jejunal motility in diarrhoea-predominant irritable bowel syndrome. Scandinavian Journal of Gasterenterology 31, 581-589.

Scott SM, Picon L, Knowles CH, Forquet F, Yazaki E, Williams NS, Lunniss PJ \& Wingate DL (2003) Automated quantitative analysis of nocturnal jejunal motor activity identifies abnormalities in individuals and subgroups of patients with slow transit constipation. American Journal of Gastroenterology $\mathbf{9 8}$ $1123-1134$.

Silk DB (1998) Patients' views on IBS. International Journal of Gastroenterology 3, 20-22.

Silk DBA (1995a) Clinical presentation of IBS. IBS Monitor 1 4-6.

Silk DBA (1995b) A logical approach to IBS. IBS Monitor 2, $2-4$. 
Silk DBA (2001) Impact of irritable bowel syndrome on personal relationships and working practices. European Journal of Gastroenterology and Hepatology 13, 1327-1332.

Silk DBA (2003) Management of IBS: start of a new era? European Journal of Gastroenterology and Hepatology 15, 679-696.

Small PK, Loudon MA, Hau CM, Noor H \& Campbell FC (1997) Large-scale ambulatory study of postprandial jejunal motility in irritable bowel syndrome. Scandinavian Journal of Gastroenterology 32, 39-47.

Smith VV, Lake BD, Kamm MA \& Nicholls RJ (1992) Intestinal pseudo-obstruction with deficient smooth muscle alpha fetoprotein-actin. Histopathology 21, 535-542.

Spiller RC, Trotman IF, Adrian TE, Bloom SR, Misiewicz JJ \& Silk DBA (1988) Further characterization of the 'ileal brake' reflex in man - Effect of ileal perfusion of partial digests of fat, protein and starch on jejunal motility and release of neurotension, enteroglucagen and peptide YY. Gut 29, 1042-1051.

Spiller RC, Trotman IF, Higgins BE, Ghatei MA, Grimble GK, Lee YC, Bloom SR, Misiewicz JJ \& Silk DBA (1984) The ileal brake - inhibition of jejunal motility after ileal fat infusion in man. Gut 25, 365-374.

Stanghellini V, Camilleri M \& Malagelada JR (1987) Chronic idiopathic intestinal pseudo-obstruction: clinical and intestinal manometric findings. Gut 28, 5-12.
Thompson WG, Longstreth GF, Drossman DA, Heaton KW, Irvine EJ \& Muller-Lissner S (1999) Functional bowel disorders and functional abdominal pain. Gut 45, 1143-1147.

Trotman IF \& Price CC (1986) Bloated irritable bowel syndrome defined by dynamic $99 \mathrm{mTc}$ brain scan. Lancet ii, 364-366.

Verne GN, Eaker EY, Hardy E \& Sninsky C (1995) Effect of octreotide and erythromycin on idiopathic and sclerodermaassociated intestinal pseudo-obstruction. Digestive Diseases and Sciences 40, 1892-1901.

Weber FH \& McCullum RW (1992) Clinical approaches in irritable bowel syndrome. Lancet 340, 1447-1451.

Whorwell PJ, Lupton EW, Erduran D \& Wilson K (1986a) Bladder smooth muscle dysfunction in patients with irritable bowel syndrome. Gut 27, 1014-1017.

Whorwell PJ, McCallum M, Greed FH \& Roberts CT (1986b) Non colonic features of irritable bowel syndrome. Gut 27, 37-40.

Wingate D, Hongo M, Kellow J, Lindberg G \& Smout A (2002) Disorders of gastrointestinal motility: towards a new classification. Journal of Gastroentrology and Hepatology 17, Suppl. S1-S14.

Wolfe F, Smythe HA, Yunus MB, Bennett RM, Bombardier C, Goldenberg DI et al. (1990) The American College of Rheumatology 1990 criteria for the classification of fibromyalgia. Report of the Multicenter Criteria Committee. Arthritis and Rheumatism 33, 160-172. 